these to administration of regular, rather than "as required", medication). The controlled drug timings were altered slightly so that, instead of being done by the nurses at the end of a shift, they were administered by nurses at the start of their shift when they were less tired. Since changing to the slightly different times there have been 4 reported errors relating to controlled drugs in 6 months, none of which relate to administration of controlled drugs.

Conclusion Maintaining a 12 hour gap between controlled drug administration but giving the medicine out at the start of nursing shifts has been a free and effective way to reduce the number of controlled drug errors occurring on the ward and reduce administration errors to zero.

\section{P 115 CHANGING THE TIMES OF ADMINISTRATION OF CONTROLLED DRUGS REDUCES ERRORS}

Eleanor Grogan, Leanne Hale, Dawn White, Marie Duke. Wansbeck Palliative Care Unit, Northumbria Healthcare NHS Foundation Trust, Ashington, UK

10.1136/bmjspcare-2014-000654.156

Background An innovative new palliative care unit opened in December 2011 within an NHS hospital with a completely new team, many of whom had not worked in a predominantly palliative setting before. A high volume of controlled drugs was given to patients and the number of errors, as reported by the electronic internal error reporting system, was higher than desired. Controlled drugs were given out to patients at the end of the nursing night shift (for morning drugs) and end of the late shift (for evening drugs). Changing the times of administration of drugs so that this was done at the start rather than the end of a shift was thought to offer a possible solution to this problem.

Aim To reduce the number of errors made with controlled drug administration in a cost effective way.

Result Before changing the timing of the controlled drug rounds there were 23 errors relating to controlled drugs reported in 13 months ( 7 relating to administration and 5 of 